\title{
The Effects Of Fabricational Variations On Quantum Wire Laser Gain Spectra And Performance
}

Hal Zarem, Kerry Vahala, Amnon Yariv

Hal Zarem, Kerry Vahala, Amnon Yariv, "The Effects Of Fabricational Variations On Quantum Wire Laser Gain Spectra And Performance," Proc. SPIE 1043, Laser Diode Technology and Applications, (22 June 1989); doi: 10.1117/12.976370 


\title{
The effects of fabricational variations on quantum wire laser gain spectra and performance
}

\author{
Hal Zarem, Kerry Vahala, and Amnon Yariv \\ Department of Applied Physics \\ California Institute of Technology \\ Pasadena, California 91125
}

\begin{abstract}
The effects of fabricational variations on the gain spectra of quantum wires are calculated within the limits of first order perturbation theory. Gain spectra and density of states for $50 \AA$ radius and $150 \AA$ radius cylindrical quantum wires are calculated and plotted for several different fabrication tolerances. The wave functions for a finite, cylindrical potential are calculated and a quasi-critical radius, below which the carriers are weakly confined by the potential, is established. This sets a lower limit on quantum wire size. Upper limits on the size of quantum wells, quantum wires, and quantum boxes are also discussed. The threshold current and differential gain of quantum wire lasers and quantum wire array lasers are calculated. These calculations indicate a possible reduction in threshold current of one to two orders of magnitude as compared to the best quantum well lasers to date.
\end{abstract}

\section{Introduction}

In recent years it has been shown both theoretically and experimentally that quantum well lasers have many advantages over conventional lasers. These advantages, such as ultra-low threshold current [1], narrow linewidth [2,3], reduced temperature dependence of the threshold current[4], and high modulation rate $[2,5]$, are related to the two dimensional nature of the electrons in quantum wells. As the dimensionality is reduced from three to two, the density of states acquires a sharp edge on the low energy side, increasing the number of states near the band edge. When these states are filled the gain spectrum is narrower. This is advantageous since we are generally concerned with peak gain and carriers contributing to gain in other spectral regions are wasted. As the dimensionality is further reduced to one or zero dimensions, giving quantum wires and quantum boxes, the density of states becomes even sharper and narrower. Theoretical investigations of the one dimensional or zero dimensional structures, quantum wires and quantum boxes, have predicted further enhancements in many of these areas $[2,4,6]$. Gain calculations for quantum wires and quantum boxes predict large reductions in the threshold current. These calculations also indicate that such structures will have a higher differential gain leading to higher modulation rates. There have been several observations of quantum size effects in quantum wires and quantum boxes $[7,8,9,10]$, but these structures are quite difficult to fabricate. Quantum well structures in high magnetic fields have been used to simulate some of these effects $[2,11]$.

The technology used to fabricate quantum wells relies on growth techniques capable of atomic layer tolerances, leading to actual quantum well devices which exhibit nearly ideal properties. Confinement in directions other than the growth direction requires lithographic patterning and the best lithographic techniques have resolutions on the order of ten nanometers. Quantum size effects are extremely sensitive to the dimensions of the confining structure. For these reasons, it is important to consider the effects of fabricational inhomogeneities on quantum wire and quantum dot structures. Variations in the size and shape of quantum wires or quantum dots will smear some of the sharpness out of the density of states of these structures, reducing some of the benefits of lower dimensionality. Inhomogeneities in quantum boxes have been considered recently and it was found that quantum box arrays with realistic fabrication tolerances are not well suited to high gain applications, but they may make possible very low threshold current lasers and may lead to optical amplifiers with improved noise characteristics [12]. A critical radius, below which no bound states exist for the quantum box, was also shown. 
In this paper, we investigate the properties of quantum wires, in particular, the effects of inhomogeneities on the density of states function and on gain are examined. The inhomogeneities are treated as a perturbation to an ideal wire and the perturbation energy is calculated to first order. The density of states for an array of wires with different widths is calculated and is used to calculate the gain of such a structure. This is done for cylindrical wires of $50 \AA$ radius and $150 \AA$ radius with several different degrees of inhomogeneity. The properties of a quantum wire laser are examined and we attempt to answer the question of whether quantum wires with realistic fabrication tolerances can fulfill the expectations of lower threshold currents, and higher modulation rates. The finite barrier quantum wire is studied and a quasi-critical radius is established, below which, the carriers are weakly confined by the wire. Upper limits on the wire radius, determined by the requirement that the energy subbands be separated by an energy greater than $k_{B} T$, are also discussed.

\section{Solution to the Two Dimensional Finite Well Problem}

A calculation of the electron and hole wave-functions is necessary to obtain the position of the energy subbands. In this paper we have treated the case of a cylindrical wire rather than the, somewhat simpler, rectangular wire employed elsewhere. This potential is chosen since the Schroedinger equation can be separated in the case of a finite cylindrical potential but not for a finite rectangular potential. A proper treatment of the finite potential is necessary to investigate the possibility of a critical radius below which no bound states exist. Such a critical radius has been shown to exist for the quantum box [12]. In this section we calculate the electron and hole wave-functions using the effective mass approximation. Their behavior as the radius of the wire goes to zero is examined.

In cylindrical coordinates the potential takes the simple form

$$
V=V(\rho)= \begin{cases}0 & \text { for } \rho<\rho_{0} \\ V_{0} & \text { for } \rho \geq \rho_{0}\end{cases}
$$

For this potential the Schroedinger equation is easily solved, giving

$$
\Psi(\rho, \phi, z)=N \begin{cases}J_{\nu}(k \rho) e^{i \nu \phi} e^{i k_{z} z} & \text { for } \rho<\rho_{0} \\ A K_{\nu}(\kappa \rho) e^{i \nu \phi} e^{i k_{x} z} & \text { for } \rho \geq \rho_{0}\end{cases}
$$

where $J_{\nu}(k \rho)$ is the Bessel function of order $\nu=0, \pm 1, \pm 2, \ldots$, and $K_{\nu}(\kappa \rho)$ is the modified Bessel function. The energy is given by

$$
E=\frac{\hbar^{2}}{2 m}\left(k_{\nu n}^{2}+k_{z}^{2}\right)
$$

The constants $\mathrm{N}, \mathrm{A}, k_{\nu n}$, and $\kappa_{\nu n}$ are determined by the normalization, the boundary condition at $\rho=\rho_{0}$, and the condition

$$
k_{\nu n}^{2}+\kappa_{\nu n}^{2}=\frac{2 m}{\hbar^{2}} V_{0}
$$

which comes directly from the Schroedinger equation. The radial momentum, $k_{\nu n}$, is a discrete variable whereas the axial momentum, $k_{z}$, varies continuously to fill the energy spectrum. In accordance with convention [13], $k_{\nu n}$ is the $n$th $\mathrm{k}$-value to satisfy the boundary conditions for $J_{\nu}$. In general there are an infinite number of $\mathrm{k}$-values for each $\nu$. In this treatment, only the first eight $\mathrm{k}$-values are considered. This can be done with no loss of generality since we will only populate the first two or three subbands.

There is no known way to solve for $k_{\nu n}$ analytically but for $V_{0} \gg \hbar^{2} k_{\nu n}^{2} / 2 m$, the boundary condition $J_{\nu}\left(k \rho_{0}\right)=0$ applies approximately and the zeros of $J_{\nu}$ are tabulated [13]. As the radius of the wire becomes smaller, the subband energy is pushed up towards the top of the potential well and the above approximation is no longer valid. For small radius wires, the value of $k_{\nu n}$ can be obtained by expanding $J_{\nu}(k \rho)$ and $K_{\nu}(\kappa \rho)$ about the origin. These expansions plus the continuity condition at $\rho=\rho_{0}$ give,

$$
k \rho_{0}=\left[\frac{2}{\frac{1}{2}-\ln \kappa \rho_{0}}\right]^{\frac{1}{2}}
$$

The intersection of the two curves defined by Eq. 4 and Eq. 5 gives the eigenvalues for $k$ and $\kappa$. Analysis of these two equations shows that there always is a bound state for this potential, but there exists a quasi-critical 
radius given by

$$
\rho_{c}=\frac{1}{2} \sqrt{\frac{\hbar^{2}}{2 m V_{0}}},
$$

below which the electron or hole is very weakly confined. At this point it becomes improbable that the carrier remains in the wire. For quantum boxes, there exists a strict critical radius below which no bound states exist [12]. The quasi-critical radius for quantum wires is a factor of $\pi$ smaller than the critical radius for quantum boxes.

\section{Roughened Cylinders}

In this section we treat the case of an imperfect quantum wire. The wire is taken to have hard boundaries with a potential step equal to $V_{0}$ but the potential is no longer a function only of $\rho_{0}$, but of all three coordinates. We assume that the wire is close enough to cylindrical that the roughness may be treated as a perturbation of the form,

$$
W(\rho, \phi, z)= \begin{cases}-V_{0} & \text { for } \rho_{0}<\rho \leq \rho_{0}+\delta \rho(\phi, z) \\ 0 & \text { otherwise }\end{cases}
$$

where $\delta \rho(\phi, z)$ is an arbitrary, nonnegative function of $\phi$ and $z$ whose magnitude is much less than $\rho_{0}$. We further require that $\delta \rho$ vary on a scale which is smaller than the coherence distance of the electron. With this perturbation, the average radius of the cylinder is increased by an amount $\langle\delta \rho(\phi, z)\rangle_{\phi, z}$ where \langle\rangle$_{\phi, z}$ denotes a spacial average over the coordinates $\phi$ and $z$. It is straightforward to show that, in the limit as $V_{0}$ goes to infinity, the application of first order perturbation theory to the ground state gives,

$$
<\delta E>\equiv<\Psi_{0}|W| \Psi_{0}>=-2 \frac{<\delta \rho>_{\phi, z}}{\rho_{0}} E_{01}
$$

where,

$$
E_{01}=\frac{k_{01}^{2} \hbar^{2}}{2 m}=\frac{x_{01}^{2} \hbar^{2}}{2 m \rho_{0}^{2}}
$$

is the energy of the first subband for the infinite barrier cylinder. To first order, the effect of the roughness is only through a change in the average radius. This implies that, to first order, quantum wires are insensitive to inhomogeneities that do not effect the average radius of the structure. Therefore, a fabrication tolerance of ten angstroms may be quite tolerable if the average radius does not change by more than a few angstroms from wire to wire. This does not apply if the perturbation varies with $z$ on a scale which is large compared to the coherence length of the electron. Sections of the wire which are at least one coherence length apart can be considered as separate wires so that the averaging of $\delta \rho$ should be done for $z_{0}<z<z_{0}+l_{c}$ where $l_{c}$ is the coherence length of the electron. If we assume an intraband scattering time of $2 \times 10^{-13} s[6]$ and thermal velocity, $l_{c}$ is on the order of $800 \AA$ at room temperature.

Equations 8 and 9 are general results in that they can be shown to hold for quantum wells and quantum boxes as well (with $x_{01}^{2}$ replaced by the appropriate value for wells or boxes) [12]. In the case of wells and boxes the confinement energy, $E_{10}$, is different, however. The confinement energy increases as the number of confined dimensions increases. For this reason, inhomogeneities will affect quantum boxes more severely than quantum wires and, likewise, quantum wires more severely than quantum wells. As the size of the structure is increased the confinement energy decreases, reducing the effect of inhomogeneities. The size can be increased only so far since the subbands must be separated by an energy which is greater than a few $k_{B} T$ in order for quantum size effects to be realized. For all three low dimensionality structures, the subband energy has a $\rho^{-2}$ dependence. If we define $\Delta E$ as the separation between the first and second subbands we can calculate the proportionality constant between $\Delta E$ and $\rho^{-2}$. The results are shown in Table 1 where $\Delta E$ is calculated for several values of $\rho$ with $V_{0}=\infty$ and using a spherical potential for the quantum box and a cylindrical potential for the quantum wire. In Table 1 and all of the figures and calculations here, values of constants which are appropriate for $\mathrm{GaAs}$ are chosen.

From Equation 9 we see that a small change in radius $d \rho$ gives a change in subband energy

$$
d E_{01}=-2 \frac{d \rho}{\rho_{0}} E_{01}
$$


which is the same as the result from the perturbation calculation. Comparing Equations 8 and 10 we see that the effect on the subband energy of roughening the cylinders with an average roughness $\langle\delta \rho\rangle_{\phi, z}$ is equivalent to changing the wire width by $d \rho=\langle\delta \rho\rangle_{\phi, z}$. It should be noted that Equation 10 is an upper limit since it applies for the case of an infinite barrier; the finite barrier case will produce a smaller shift because of the softer boundaries. In what follows we assume that we have an ensemble of wires with different values of $\langle\delta \rho\rangle_{\phi, z}$. In accordance with the central limit theorem we assume a Gaussian distribution of wire radii

$$
P(\rho)=\frac{1}{\sqrt{2 \pi} \delta \rho} \exp \left[\frac{-\left(\rho-\rho_{0}\right)^{2}}{\delta \rho^{2}}\right]
$$

where $\rho_{0}$ is the average wire radius and $\delta \rho$ is the standard deviation of $\langle\delta \rho\rangle_{\phi, z}$. The length of a typical semi-conductor laser is several hundred times the coherence length of the electron so the gain of a single wire must be obtained by considering the gain of many wires each of length $l_{c}$.

\section{Gain Spectra}

The density of states for an ideal (unroughened) wire of radius $\rho_{0}$ is,

$$
D(E)=\sum_{l=1}^{\infty} \frac{\left(m_{r} / 2 \hbar^{2}\right)^{1 / 2}}{\pi^{2} \rho_{0}^{2}}\left(E-E_{g}-E_{l}\right)^{-1 / 2} \eta_{l}
$$

where $E$ is the energy of the transition, $E_{g}$ is the band-gap, $E_{l}$ is the position of the $l^{\text {th }}$ subband (the index $l$ is a combination of the indices $n$ and $\nu$ of the previous sections), $\eta_{l}$ is the degeneracy of the $l^{\text {th }}$ subband ( $\eta_{l}=1$ for $\nu=0, \eta_{l}=2$ otherwise) and $m_{r}=m_{e} m_{h} /\left(m_{e}+m_{h}\right)$ where $m_{e}$ and $m_{h}$ are the masses of the electron and the hole respectively. In all of the calculations here, values of the material parameters which are appropriate for GaAs are used. These values are: $m_{e}=0.067, m_{h}=0.45, E_{g}=1.424$. The subband positions, $E_{l}$, are calculated assuming an infinite bandgap discontinuity.

Changing the radius of a wire will affect the gain by moving the subband edge. We obtain a Gaussian distribution of subband energies by combining Equations 10 and 11

$$
P\left(E_{l}\right)=\frac{1}{\sqrt{2 \pi} \delta E_{l}} \exp \left[\frac{-\left(E_{l}-\overline{E_{l}}\right)^{2}}{\delta E_{l}^{2}}\right]
$$

where $\delta E_{l}$ and $E_{l}$ are related to $\delta \rho$ and $\rho$ through Equations 8 and 9 . The bulk density of states for the material is found by integrating Equation 12 over all values of $E_{l}$ weighted by Equation 13 giving the inhomogeneous density of states,

$$
\begin{array}{r}
D_{i n h}(E)=\sum_{l=1}^{\infty} \frac{1}{\delta E \sqrt{2 \pi}}\left(\frac{m_{r}}{2}\right)^{1 / 2} \frac{\eta_{l}}{\pi \rho_{0}^{2} \hbar} \int\left(E-E_{g}-E_{l}\right)^{-1 / 2} \\
\exp \left[\frac{-\left(E_{l}-\overline{E_{l}}\right)^{2}}{\delta E_{l}^{2}}\right] d E_{l}
\end{array}
$$

The integral in Equation 14 is evaluated numerically and the inhomogeneous density of states is plotted for wires of radius $\rho=50 \AA$ in Figure 1 . The effects of the inhomogeneities are quite dramatic: for $\delta \rho=2.5 \AA$, which corresponds to a mono-layer variation in radius, the subbands are distinct and the density of states resembles the ideal density of states, whereas, for $\delta \rho=10 \AA$ all subband structure is washed out and the density of states resembles that of bulk material. As the subbands broaden, their peak value decreases since the area under each curve must be the same. In general, the magnitude of the density of states will be inversely proportional to the radius of the wire if $\delta \rho / \rho$ is kept fixed (this follows directly from Equation 14 when the dependence of $E_{l}$ and $\delta E_{l}$ on $\rho_{0}$ is considered). For $\rho=150 \AA$ the first and second subband are separated by only $18 \mathrm{meV}$. This means that at room temperature there will be substantial filling of the second subband when the first subband is partially filled, leading to increased threshold currents.

The density of states calculations are the basis of the gain calculations to follow. The broadening caused by the inhomogeneities is, in all cases considered here, greater than or equal to relaxation broadening. The gain is therefore given by,

$$
G(E)=\frac{E}{\hbar} \sqrt{\mu / \epsilon}|d|^{2} D(E)\left(f_{c}-f_{v}\right)
$$




\begin{tabular}{|c|c|c|c|}
\hline & \multicolumn{3}{|c|}{$\Delta E(m e V)$} \\
\hline & Welt & Wre & Box \\
\hline & $\hbar^{2}$ & $\hbar^{2}$ & $\hbar^{2}$ \\
\hline$\rho=\rho_{0}$ & $(1.4) \overline{2 \mathrm{~m} \rho_{0}^{2}}$ & $\int^{(8.0)} \overline{2 m \rho_{0}^{2}}$ & $\sqrt{20.3)} \overline{2 \mathrm{~m} \rho^{2}}$ \\
\hline cb & 168 & 202 & 234 \\
\hline $\int_{\text {Vb }}^{p=00 x^{\prime}}$ & 25 & 30 & 35 \\
\hline cb & 42 & 51 & 50 \\
\hline${ }_{\text {vb }}^{\rho=100 \lambda}$ & 6.2 & 7.6 & 8.8 \\
\hline cb & 11 & 13 & 15 \\
\hline vo & 1.6 & 1.0 & 2.2 \\
\hline
\end{tabular}

Table 1: The separation of the first and second subbands for different size quantum wells, quantum wires, and quantum boxes in GaAs. The parameter $\rho$ is the radius, or, in then case of quantum wells, a half width. When $\Delta \mathrm{E}$ is smaller than $k_{B} T$, thermal broadening smears out quantum size effects.

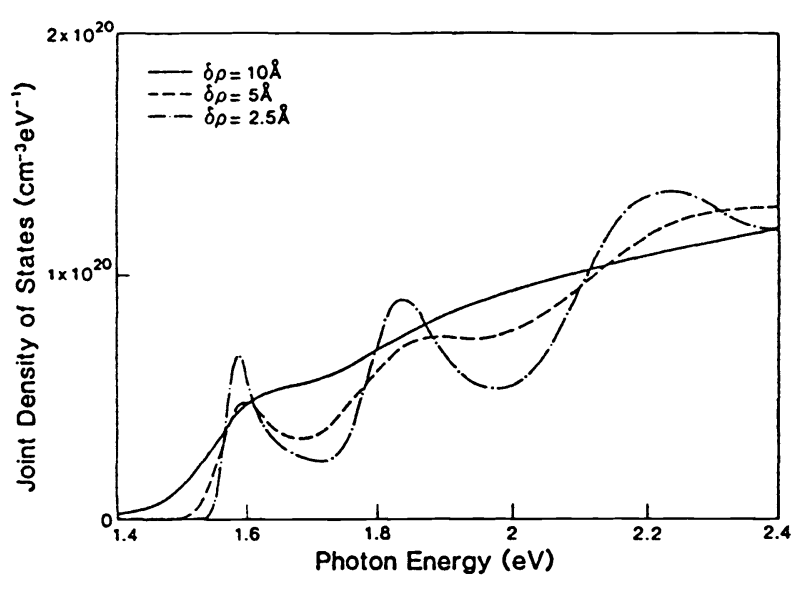

Figure 1: The density of states for $50 \AA$ radius quantum wires with three different values of the roughness parameter; $\delta \rho=2.5 \AA, \delta \rho=5.0 \AA$, and $\delta \rho=10.0 \AA$.

where $\epsilon$ is the dielectric constant of the material, $\mu$ is the magnetic susceptibility, $f_{c}$ and $f_{v}$ are the Fermi distributions for the conduction and valence bands respectively, and $d$ is the component of the dipole moment parallel to the electric field. A value of $d / q=4 \AA \mathrm{h}$ as been assumed and any dependence of $d$ on the orientation of the electric field with respect to the wire axis is ignored. The light hole band has also been ignored in the calculation. The overlap of the field with the gain region is not yet accounted for in these plots. As the carrier density is doubled, the peak gain increases almost proportionally, indicating that, even at this high carrier density, the carriers are going predominantly into the first subband. In Figure 2 gain is calculated for the density of states functions of Figure 1, showing the effects of increased inhomogeneities on the gain spectrum. All of the curves are for the same carrier density. Here, the benefits of a sharp density of states function are clear as the peak gain drops by roughly a factor of two when $\delta \rho$ goes from $2.5 \AA$ to $10 \AA$. Figure 3 shows the dependence of peak gain on carrier density for these same wires. The gain rises steeply at first, but it begins to level off at around $7 \times 10^{18} \mathrm{~cm}^{-3}$ as the first subband becomes full. At a carrier density of roughly $1.3 \times 10^{18} \mathrm{~cm}^{-3}$ the gain begins to rise sharply once again. It is at this point that the gain of the second subband exceeds that of the first. This has been observed experimentally in quantum well lasers [14] and it is accompanied by a large change in lasing wavelength. A plot similar to that of Figure 3 but for $150 \AA$ radius wires is shown in Figure 4 . For any given carrier density, the gain of a $150 \AA$ wire is lower than that of a $50 \AA$ wire. This is due to two factors: first, the density of states is smaller for larger wires, and second, the subbands are separated by an energy which is less than $k_{B} T$ so several subbands are being filled simultaneously.

\section{Lasing Properties}

So far, we have discussed the properties of quantum wires without considering the device in which they are to be imbedded. In this section ve consider the modal gain, threshold current, and the modulation rate for a quantum wire laser.

Figure 5 shows a schematic diagram of a quantum wire array laser. The dashed cylinder which envelops the quantum wires represents the optical confinement region and the width of the optical mode in the $\mathbf{x}$ and $y$ directions is shown as $W_{x}$ and $W_{y}$. The modal gain is obtained by multiplying the bulk gain for a wire by the confinement factor, which is a measure of the overlap of the optical field with the gain region. Switching to rectangular coordinates and keeping the cylinder axis as the z-axis, the confinement factor is a product of confinement factors for the $x$ and $y$ directions, $\Gamma_{x}=d / W_{x}$ and $\Gamma_{y}=d / W_{y}$. For a single wire of $50 \AA$ radius, 


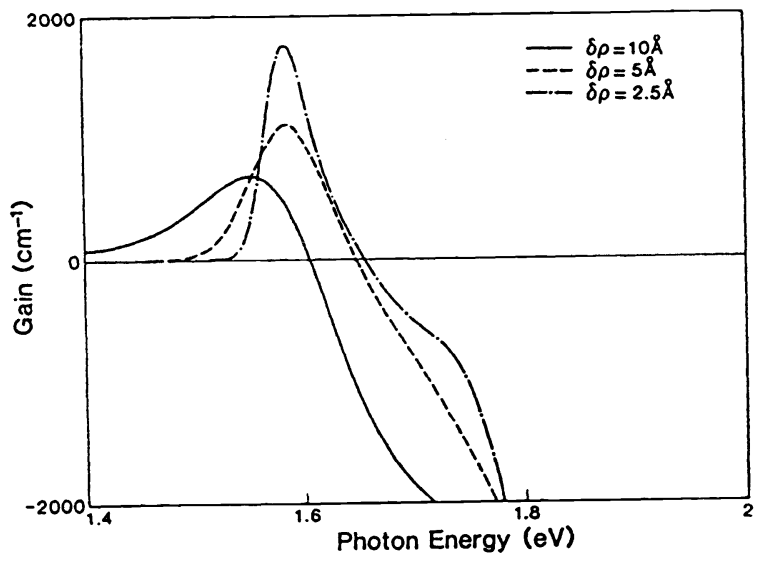

Figure 2: Gain as a function of photon energy for $50 \AA$ radius quantum wires with $\delta \rho=2.5 \AA, \delta \rho=$ $5.0 \AA$, and $\delta \rho=10.0 \AA$ at a carrier concentration of $4 \times 10^{18} \mathrm{~cm}^{-3}$.

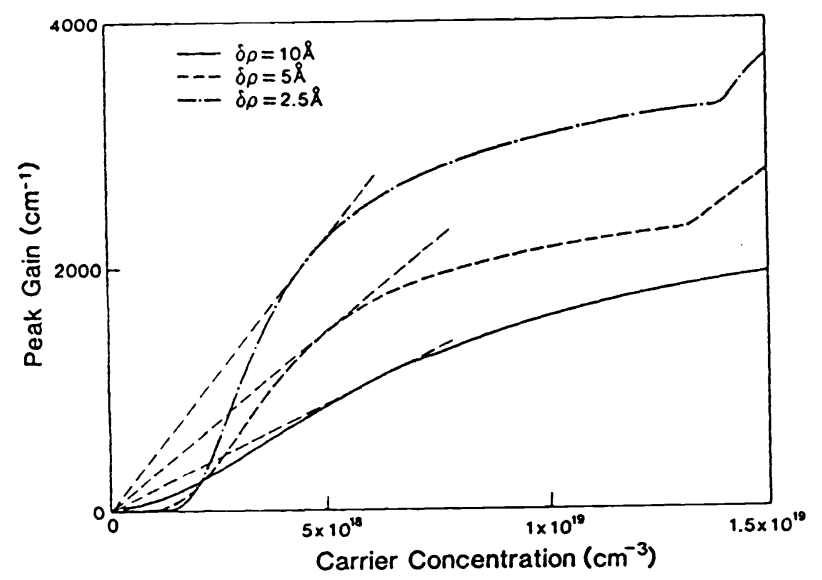

Figure 3: Peak gain as a function of carrier concentration for the quantum wires of Figure 1 . The sudden change in slope at high carrier densities is indicative of second quantized state lasing. The point of maximum gain per carrier density is given by the dashed tangent.

$\Gamma_{x}=\Gamma_{y}=0.04$ is a reasonable value [1]. If we take a peak gain of $3000 \mathrm{~cm}^{-1}$ and the above confinement factors, the peak modal gain is $G_{\text {mode }}=4.8 \mathrm{~cm}^{-1}$ per wire. This is about enough gain to overcome the distributed losses, so clearly we must employ an array of such quantum wires to make a laser.

The threshold gain is given by,

$$
G_{\text {modal,th }}=\alpha-\frac{1}{l} \ln R
$$

where $\alpha$ is the distributed loss coefficient for the mode, $l$ is the length of the cavity, and $R$ is the reflectivity of the mirrors. To estimate threshold current densities we assume values of $\alpha=3 \mathrm{~cm}^{-1}, l=300 \mu \mathrm{m}$, and $R=0.9$. For these values, $G_{\text {modal, } t h}=6.5 \mathrm{~cm}^{-1}$. To achieve the lowest threshold current, we want to pump the wires to the point of maximum gain per carrier density. This is the point on the peak gain vs. carrier density curve whose tangent intersects the origin. From Figure 3, we see that this occurs at a carrier density of approximately $5 \times 10^{18} \mathrm{~cm}^{-3}$ for a $50 \AA$ radius wire with $\delta \rho=2.5 \AA$. At this point, the gain in the wire is approximately $2250 \mathrm{~cm}^{-1}$. To optimize the structure for low threshold current operation, the number of wires in the array should be chosen such that at threshold they are pumped to this point. With the above confinement factors, a laser with two such wires would have a modal gain of $7.2 \mathrm{~cm}^{-1}$, which is just above the estimated threshold gain. To arrive at a threshold current, a value of the carrier lifetime must be assumed. The effect of the two dimensional confinement on the carrier lifetime is not known, so bulk the carrier lifetime is used. If we assume a carrier lifetime of $3 n s$ [15], and 100 percent injection efficiency, this two wire laser would have a threshold current of approximately $11 \mu \mathrm{A}$, which is nearly two orders of magnitude lower than the best quantum well lasers. When the same considerations are applied to a $50 \AA$ wire with $\delta \rho=10 \AA$, the optimal carrier density is approximately $6.5 \times 10^{18} \mathrm{~cm}^{-3}$, and a laser containing four such wires would have a threshold current of approximately $33 \mu \mathrm{A}$, which is still extremely low. In the case of $150 \AA$ radius wires, the confinement factors increase to $\Gamma_{x}=\Gamma_{y}=0.12$. Working backwards, if we assume a modal gain of $6.5 \mathrm{~cm}^{-1}$ and the above confinement factors, the bulk gain requirement is only $450 \mathrm{~cm}^{-1}$. One wire can provide this gain and, under the same assumptions as above, the estimated threshold current is $34 \mu \mathrm{A}$ for the cases of $\delta \rho=15 \AA$ and $\delta \rho=30 \AA$ (here, the effects of thermal broadening exceed those of the inhomogeneities so both $\delta \rho=15 \AA$ and $\delta \rho=30 \AA$ wires behave the same at room temperature). It is clear that the threshold current does not suffer much as the fabrication tolerance requirements are relaxed.

The modulation bandwidth is determined by the relaxation oscillation corner frequency,

$$
f_{c}=\frac{1}{2 \pi}\left[\frac{G^{\prime} P_{0}}{\tau_{p}}\right]^{1 / 2}
$$




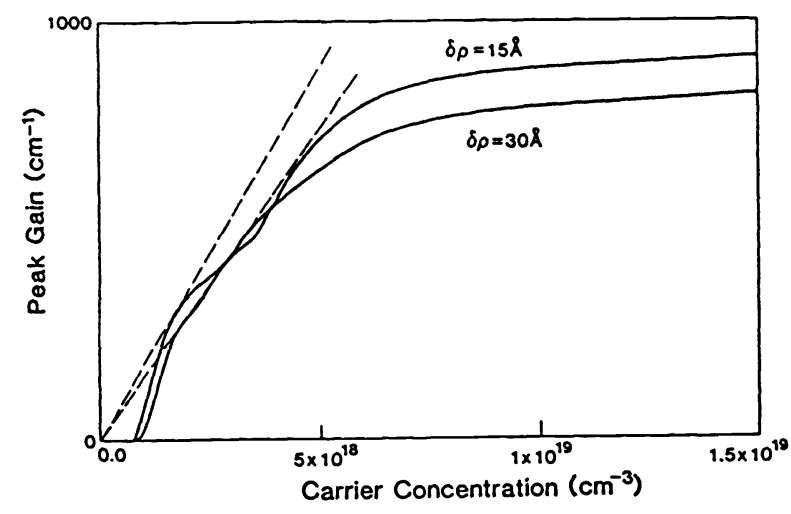

Figure 4: Peak gain as a function of carrier concentration for $\rho=150 \AA$ quantum wires. The point of maximum gain per carrier density is given by the dashed tangent.

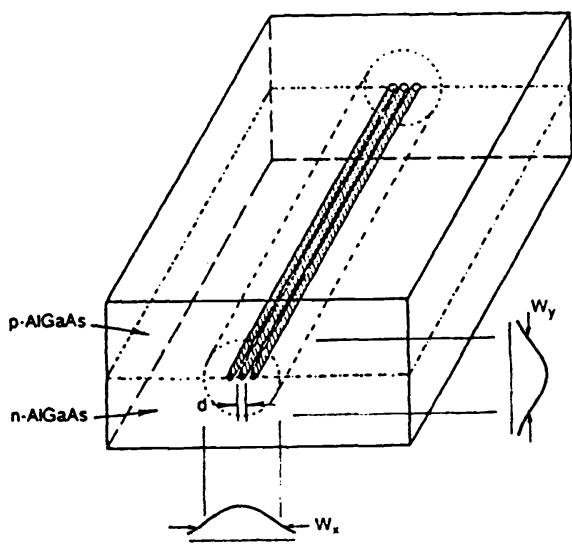

Figure 5: A schematic of a quantum wire array laser. The width of the optical mode is given by $W_{x}$ and $W_{y}$ and the wire diameter is $d$. The confinement factor is given by the relation $\Gamma_{i}=d / W_{i}$.

where $G^{\prime}=d G / d n$ is the differential gain , $P_{0}$ is the steady state photon density in the cavity, and $\tau_{p}$ is the photon lifetime [16]. Due to the sharpened density of states function, the differential gain for quantum wires should be higher than for bulk material. The differential gain can be found from Figures 3 and 4 . For the $50 \AA$ radius wires, the maximum differential gain is, $d G / d n=1.0 \times 10^{-15} \mathrm{~cm}^{2}$ and $2.2 \times 10^{-16} \mathrm{~cm}^{2}$ for $\delta \rho=2.5 \AA$ and $10 \AA$ respectively. This is to be compared to a value of $d G / d n=2.0 \times 10^{-16} \mathrm{~cm}^{2}$ for bulk GaAs, and $d G / d n=5.0 \times 10^{-16} \mathrm{~cm}^{2}$ for quantum wells [2] The differential gain for the $150 \AA$ wires, as calculated from Figure 4, is close to the bulk value. We see that the modulation bandwidth of the well fabricated wire is greater by a factor of $\sqrt{5}$ than that of a laser with a bulk active layer, but that for more realistic fabrication tolerances the increased bandwidth disappears.

\section{Conclusion}

We have calculated the gain spectra of quantum wires accounting for fabricational inhomogeneities. The inhomogeneities were treated as a perturbation and it was found that to first order, the component of the perturbation which varies quickly compared to the coherence length of the carrier is averaged out and has no effect if the wire radius is chosen so that the roughness function has zero average. This indicates that the gain in quantum wires is somewhat insensitive to small scale inhomogeneities. An ensemble of wires with differing widths was considered and a bulk density of states and gain were calculated from this. According to these calculations, quantum wires with realistic fabrication tolerances are advantageous for low threshold laser applications but unless they are fabricated with atomic layer precision, they will not display a large enhancement in modulation bandwidth.

The wave functions for quantum wires have been examined using a finite cylindrical potential and we found a quasi-critical radius, below which, the carriers are not confined by the potential, although, in a strict sense, the state is a bound state. This puts a lower limit on the radius of quantum wires. An upper limit on the wire size is given by the requirement that the subbands be separated by an energy greater than $k_{B} T$ and the effects of increasing the size and changing the number of quantized dimensions were tabulated for two, one, and zero dimensional structure.

As a result of these calculations, it is concluded that quantum wire lasers with realistic fabrication tolerances are promising structures for reduced threshold current. Reductions of one to two orders of magnitude over the best quantum well lasers are possible. Such large reductions in threshold current could open new realms of applications for semiconductor lasers. 
Two of us (H.Z. and A.Y.) would like to acknowledge the support of ONR and DARPA. One of us (K.V.) would like to acknowledge the support of ONR.

\section{References}

[1] P. L. Derry, A. Yariv, K. Y. Lau, N. Bar-Chaim, and J. Rosenberg, "Ultralow-Threshold GradedIndex separate-confinement Single Quantum Well Buried Heterostructure (Al,Ga)As Lasers With High Reflectivity Coatings," Appl. Phys. Lett., vol. 50, pp. 1773-1775, 1987.

[2] Y. Arakawa, K.Vahala, and A.Yariv, "Dynamic and Spectral Properties of Semiconductor Lasers With Quantum-Well and Quantum-Wire Effects," Surf. Sci., vol. 174, pp.155-162, 1986.

[3] P. L. Derry, T. R. Chen, Y. H. Zhuang, J. Paslaski, M. Mittelstein, K. Vahala, and A. Yariv, "Spectral and Dynamic Characteristics of Buried Heterostructure Single Quantum Well (Al,Ga)As Lasers," Appl. Phys. Lett., vol. 53, pp.271-273, 1988.

[4] Y. Arakawa and H. Sakaki, "Multidimensional quantum well laser and temperature dependence of threshold current," Appl. Phys. Lett., vol. 40, pp. 939-941, 1980.

[5] K. Uomi, T. Mishima, and N. Chinone, "Ultrahigh Relaxation Oscillation Frequency (up to $30 \mathrm{GHz}$ ) of Highly P-Doped GaAs/GaAlAs Multiple Quantum Well Lasers," Appl. Phys. Lett., vol. 51, pp. 78-80, 1987.

[6] M. Asada, Y. Miyamoto, and Y. Suematsu, "Gain and the Threshold of Three-Dimensional QuantumBox Lasers," IEEE Journal of Quantum Electron., vol. QE-22, pp. 1915-1921, 1986.

[7] P. M. Petroff A. C. Gossard, R. A. Logan, and W. Wiegman, "Toward Quantum Wires: Fabrication and Optical Properties," Appl. Phys. Lett., vol. 41, pp.635-638, 1982.

[8] K. Kash, A. Scherer, J. M. Worlock, H. G. Craighead, and M. C. Tamargo, "Optical Spectroscopy of Ultrasmall Structures Etched From Quantum Wells," Appl. Phys. Lett., vol. 49, pp.1043-1045, 1986.

[9] J. Cibert, P. M. Petroff, G. J. Dolan, A. C. Gossard, and J. H. English, "Optically Detected Carrier Confinement to One and Zero Dimension in GaAs Quantum Well Wires and Boxes," Appl. Phys. Lett., vol. 49, pp.1275-1277, 1986.

[10] H. Temkin, G. J. Dolan, M. B. Panish, and S. N. G. Chu, "Low-Temperature Photoluminescence From InGaAs/InP Quantum Wires and Boxes," Appl. Phys. Lett., vol. 50, pp. 413-415, 1987.

[11] K. Vahala, Y. Arakawa, and A. Yariv, "Reduction of the Field Spectrum Linewidth of a Multiple Quantum Well Laser in a High Magnetic Field - Spectral Properties of Quantum Dot Lasers," Appl. Phys. Lett., vol. 50, pp.365-367, 1987.

[12] K. Vahala, "Quantum Box Fabrication Tolerance and size Limits in Semiconductors and Their Effect on Optical Gain," IEEE Journal of Quantum Electron., vol. QE-24, pp. 523-530, 1988.

[13] Abromawitz and Stegun, Handbook of Mathematical Functions, National Bureau of Standards, 1972.

[14] M. Mittelstein, Y.Arakawa, A. Larsson, and A. Yariv, "Second Quantized State Lasing of a Current Pumped Single Quantum Well Laser,” Appl. Phys. Lett., vol. 49, pp.1689-1691, 1986.

[15] H. C. Casey, Jr. and M. B. Panish, Heterostructure Lasers (Academic Press, New York, 1978).

[16] K. Y. Lau, N. Bar-Chaim, I. Ury, C. Harder, and A. Yariv, "11-GHz Direct Modulation Bandwidth GaAlAs Window Laser Operating at Room Temperature," Appl. Phys. Lett., vol. 45, pp. 316-318, 1984. 